\title{
Entropy and Negentropy Principles in the I-Theory
}

\author{
H. H. Swami Isa ${ }^{1}$, Christophe Dumas ${ }^{2}$ \\ ${ }^{1}$ Global Energy Parliament, Trivandrum, Kerala, India \\ ${ }^{2}$ Atomic Energy Commission, Cadarache, France \\ Email: isa@global-energy-parliament.net,christophe.dumas@cea.fr
}

How to cite this paper: Isa, H.H.S. and Dumas, C. (2020) Entropy and Negentropy Principles in the I-Theory. Journal of High Energy Physics, Gravitation and Cosmolo$g y, 6,259-273$.

https://doi.org/10.4236/jhepgc.2020.62020

Received: January 31, 2020

Accepted: March 31, 2020

Published: April 3, 2020

Copyright (c) 2020 by author(s) and Scientific Research Publishing Inc. This work is licensed under the Creative Commons Attribution International License (CC BY 4.0).

http://creativecommons.org/licenses/by/4.0/

\begin{abstract}
Applying the I-Theory, this paper gives a new outlook about the concept of Entropy and Negentropy. Using $S_{\infty}$ particle as $100 \%$ repelling energy and $\mathrm{A}_{1}$ particle as the starting point of attraction, we are able to define Entropy and Negentropy on the quantum level. As the I-Theory explains that repulsion force is driven by Weak Force and attraction is driven by Strong Force, we also analyze Entropy and Negentropy in terms of the Fundamental Forces.
\end{abstract}

\section{Keywords}

I-Theory, I-Particle, Entropy, Negentropy, Syntropy, Intelligence, Black Matter, White Matter, Red Matter, Gravitation, Strong Force, Weak Force, Free Energy

\section{Introduction}

In a previous article entitled "I-Theory-a Unifying Quantum Theory?" [1], the authors introduced the I-Theory as a unifying theory. The paper showed how I-Theory successfully encompasses all major theories like the Standard Model, General Relativity, Big Bang, Super symmetry, etc., and finally offered a new and expanded context for the main concepts in physics, like Dark Matter, Dark Energy, the four interaction forces, and so on. Since the I-Theory is a unifying theory, it is also able to elucidate the destructive and constructive forces, which are the driving forces of life.

In this context, we will explore the concepts of Entropy and Negentropy. Using the I-Theory, we will explain them in a simple manner and make a link with the gravitational force.

\section{The Concepts of Entropy and Negentropy}

The Universe is driven by cycles. A cycle is composed of two opposing princip- 
als, Entropy and Negentropy. Entropy is the more commonly known, while Negentropy is its opposite.

\subsection{Entropy}

The Entropy principle was initially introduced by the French scientist Sadi Carnot in 1824 [2]. Carnot may not have used the word "Entropy", but he developed the principle. The term entropy was introduced in 1865 by Rudolf Clausius [3] from a Greek word meaning "transformation". It was Clausius who completed the second principle of thermodynamics. Entropy characterizes the degree of disorganization, or unpredictability of the information content of a system.

In thermodynamics, Entropy is defined by the following formula:

$$
\Delta S=\frac{Q}{T}
$$

where:

- $\Delta S$ : The entropy evolution in the system.

- $Q$ : The heat exchange by the system.

- T: The system temperature.

Entropy is stated, in the second principle of thermodynamics, as spontaneously increasing in an isolated system. This means:

$$
\Delta S \geq 0
$$

But thermodynamics only describes macroscopic effects. Therefore, the Entropy principle has been extended to subatomic particles by Boltzmann. He defined Entropy as a state function [4]:

$$
S=k_{B} \ln (\Omega)
$$

where:

- $S$ is the state function Entropy.

- $k_{B}$ is the Boltzmann constant.

- $\Omega$ is the state number.

In statistical physics, the state number is defined by:

$$
\Omega=N * P
$$

where:

- $\quad N$ is the number of possible states.

- $\quad P$ is the quantity of movements.

Boltzmann has shown that Equation (3) is only an extension of Equation (1).

Entropy is directly linked to the state element. Let's consider two systems:

o System 1 is solid state. The system is a fully ordered system.

o System 2 is gas state. The system is partially ordered. An atom or a molecule can be located at any place in space.

Since the number of possible arrangements is higher in System 2 than in System 1, we can write:

$$
\Omega_{2}>\Omega_{1}
$$

According to Boltzmann's definition, we can write: 


$$
\left\{\begin{array}{l}
S_{2}=k_{B} \ln \left(\Omega_{2}\right) \\
S_{1}=k_{B} \ln \left(\Omega_{1}\right)
\end{array}\right.
$$

which means:

$$
S_{2}>S_{1}
$$

The more space a system has, the more the system is disorganized, and the greater the number of possible locations. Therefore, the Entropy is high. This is indicated in Figure 1.

Later, the Entropy principle has been used in astrophysics, especially for measuring the Black Hole thermodynamics. Starting from Stephen Hawking's work, Jacob Bekenstein guessed that the Black Hole Entropy was proportional to the area of its event horizon divided by the Planck area. Using the thermodynamic relationship between energy, temperature and Entropy, Hawking was able to confirm Bekenstein's conjecture and show that [5]:

$$
S_{B H}=\frac{k_{B} * A}{4 * l_{p}^{2}}
$$

where:

- $k_{B}$ is the Boltzmann constant.

- $A$ is the area of the event horizon.

- $l_{p}=\sqrt{G \hbar / c^{3}}$ is the Planck length.

In information theory, Entropy quantifies the lack of information. It is also known as Shannon Entropy. This is another expression of Boltzmann's Entropy in thermodynamics. Shannon Entropy is a mathematical function that intuitively corresponds to the amount of information contained or delivered by an information source [6]. This source can be a text written in a given language, an electrical signal or any computer file (byte data).

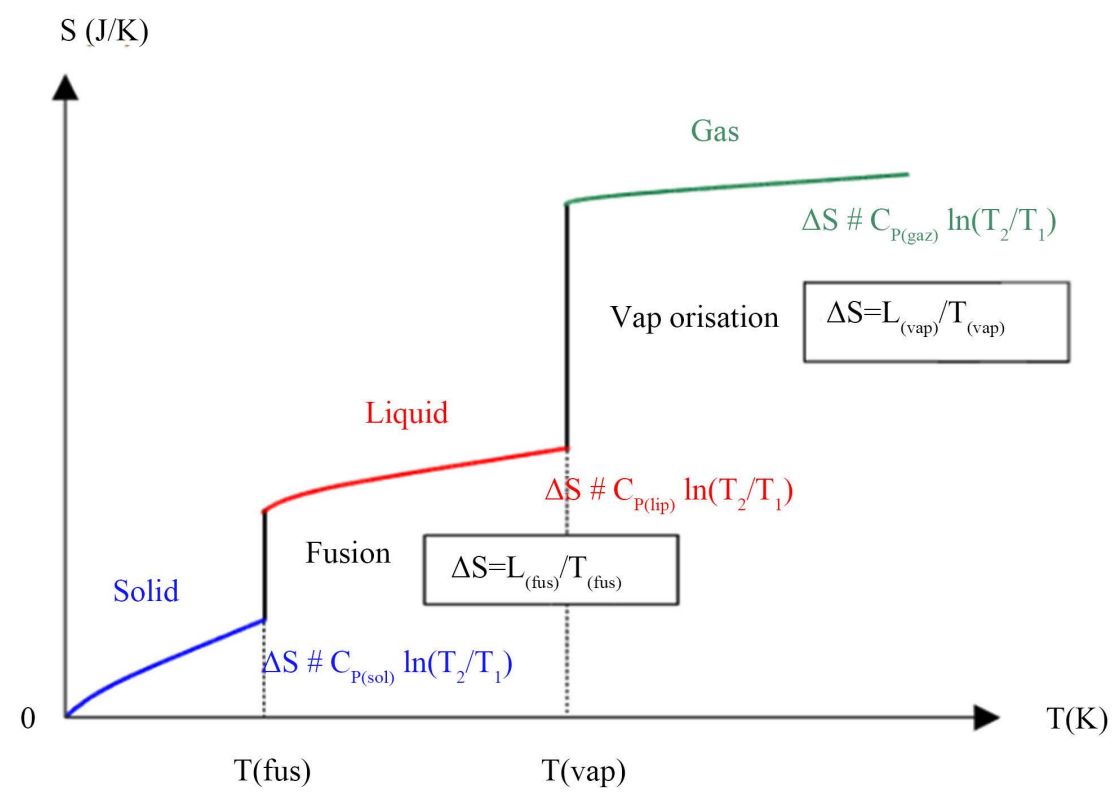

Figure 1. Entropy and state element. 
From a receiver's point of view, the greater amount of different information the source emits, the greater is the Entropy (or uncertainty about what the source emits). Thus, if a source always sends the same symbol, for example the digit "0", then its Entropy is null, which is the minimal Entropy. Certainly, a receiver that only knows the transmission statistics of the source is assured that the next symbol will be " 0 ". On the other hand, if the source sends alternatively " 0 " or "1", the receiver is uncertain about the next digit it will receive. The Entropy of the source in this case is therefore non-zero (positive) and quantitatively represents the uncertainty of the information from the source.

In ecology, Shannon Entropy is used as a measure of biodiversity through the Shannon index.

\subsection{Negentropy}

Entropy's opposing force is called Negentropy. The term Negentropy is an abbreviation of "Negative Entropy". Negentropy is also called Syntropy. It was introduced in the context of a major scientific debate by several physicists including by Erwin Schrödinger [7]. Negentropy is used to explain the presence of "order" within living beings and their tendency to oppose the chaos and disorganization that governs physical systems. Negentropy has been developed and put into perspective based on the work of mathematician Claude Shannon by Léon Brillouin [8] [9]. Negentropy does not contradict Sadi Carnot's work.

Of thermodynamic origin, Negentropy is used systemically as a synonym for cohesion strength while Entropy is a synonym for repulsion force.

Entropy is stated, in the second principle of thermodynamics, as spontaneously increasing in an isolated system. Under this condition, the principle of Negentropy is therefore necessarily limited in time or space or can only be applied to an open system.

Statistical Negentropy is closely linked to free enthalpy. In 1873, Willard Gibbs created a diagram illustrating the concept of free energy corresponding to free enthalpy [10]. Gibbs explained that a system can be characterized by its capacity for Entropy. This quantity is the amount of Entropy that may be increased without changing in internal energy or increasing its volume. In other words, it is a difference between maximum possible Entropy, under assumed conditions, and its actual Entropy. It corresponds exactly to the definition of Negentropy adopted in statistics and information theory.

Massieu introduced a similar physical quantity in 1869 for the isothermal process [11] [12]. Massieu's potential and free Entropy differs just with a figure sign. Planck has completed this for the isothermal-isobaric process [13]. More recently, the Massieu-Planck thermodynamic potential, known also as free entropy, has been shown to play a great role in the so-called entropic formulation of statistical mechanics [14], molecular biology [15] and thermodynamic non-equilibrium processes [16]. The Negentropy is expressed by:

$$
J=S_{\max }-S=-\phi=-k_{B} \ln (Z)
$$


where:

- $\quad J$ is Negentropy.

- $\quad S$ is Entropy.

- $\phi$ is the Massieu potential.

- $\quad k_{B}$ is the Boltzmann constant.

- $Z$ is the partition function.

This principle is not easy to understand. The best example of it is life itself. It is the law of order and organization, finality and differentiation. Life has the ability to attract, evolve and bring together ever-increasingly complex forms creating something new. For example, a new galaxy is formed from the ruins of an older galaxy. Individual cells come together in order to create an organism. In nature, there is a force focusing energy to create and maintain a system. This law of life is called Negentropy.

In an organism, the degree of internal disorganization is Entropy and the degree of internal organization is Negentropy. The sum of these two quantities represents the level of health of an organism in the present state and the transformation potential for the evolution of that system or organism [17] [18].

According to the principle of Entropy, all organized forms of matter require more energy than those that are less organized. These organized forms will lose their order and initial energy, unless they are constantly consuming energy. For example, plants need energy in the form of water and sunlight to grow. When that is deprived they begin to decay. Likewise, a new structure or machine will fall apart unless energy is applied to maintain it. Time after time, systems lose energy becoming less efficient allowing for disease, illness and finally death to set in. In order to overcome this natural tendency, Negentropy is an essential principle for balancing it.

\section{The I-Theory}

\subsection{Overview of the I-Theory}

The laws of Nature are subtle and still beyond our capacity to understand. The I-Theory is a unifying theory, which describes these laws comprehensively, because it starts with the fundamental building brick of nature and the Universe: the basic quantum of energy.

The I-Theory proposes a new particle, the only true elementary particle which constitutes so-called elementary particles like quarks, leptons and bosons. An "I-particle", as it is called, is vibrating, electrically polarized, and composed of three different matters. These matters are determined by the frequency and wavelength of the vibration. The I-particle's composition is $20 \%$ negative (Black Matter), 30\% neutral (Red Matter) and 50\% positive (White Matter) (Figure 2) [1].

White, Red and Black Matters have different frequencies and wavelengths. Frequency is inversely proportional to wavelength. Black Matter has the highest frequency and shortest wavelength. White Matter has the lowest frequency and 


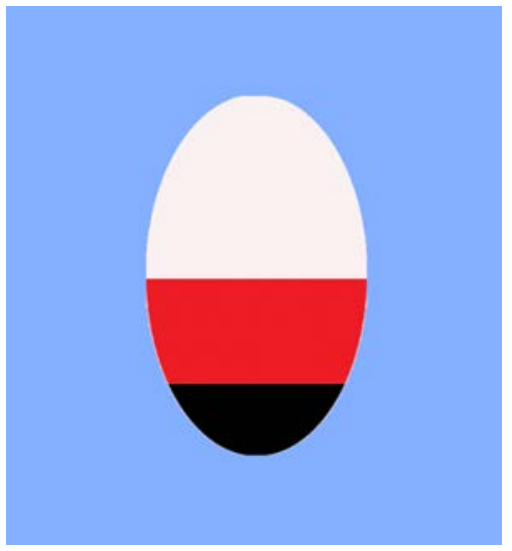

Figure 2. I-particle, showing $20 \%$ black matter, $30 \%$ red matter, $50 \%$ white matter.

longest wavelength. Red Matter has mid-level frequency and mid-level wavelength [1].

In more detail, the pattern of the I-particle is a toroid (Figure 3), like a vibrating string. The toroid's center is composed of Red Matter. The vibration propagates from the center upwards and over the top. This vibration creates White Matter (positively charged). Then the vibration propagates from the center downwards and outwards along the bottom part. This vibration creates Black Matter (negatively charged).

Since I-particles are electrically charged, they could be in attraction or repulsion state (Figure 4).

There are five elements in total, each constituted by a certain percentage of attraction and repulsion force, as given in Table 1:

The I-Theory also introduces two more particles [1]. The first one is called $S_{\infty}$ particle, which refers to a particle of space. The second one is called $A_{1}$ particle and refers to a particle of subtle air. $A_{1}$ particle is also commonly known by the name of the Higgs boson.

\subsection{I-Theory in Entropy and Negentropy}

The I-Theory gives a deeper understanding of the principles of Entropy and Negentropy.

When $100 \%$ of I-particles are in repelling state $\left(S_{\infty}\right.$ particle), space is very dominant. All the I-particles are in the same state. It means that number of possible arrangement is the highest. Considering the Equation (4), we can conclude: $\Omega_{S_{\infty}}=\operatorname{Max}(\Omega)$.

So we can write:

$$
S_{S_{\infty}}=S_{\text {Max }}
$$

When attraction starts, subtle air is formed. Taking into account that the $A_{1}$ particle's level of repelling energy is from $87.5 \%$ up to $99 \%$, space is still very dominant but less than $S_{\infty}$ particle. Therefore, we can write:

$$
\Omega_{S_{\infty}} \gg \Omega_{A_{1}}
$$


Table 1. Composition of matter by percentage of attracting and repelling I-particles.

\begin{tabular}{|c|c|c|c|c|c|c|c|c|c|c|}
\hline & Subtle Space & Gross Space & Subtle Air & Gross Air & Subtle Fire & Gross Fire & Subtle Liquid & Gross Liquid & Subtle Solid & Gross Solid \\
\hline Attraction (\%) & 0 & $0-1$ & $1-12.5$ & $12.5-25$ & $25-37.5$ & $37.5-50$ & $50-62.5$ & $62.5-75$ & $75-87.5$ & $87.5-100$ \\
\hline Repulsion (\%) & 100 & $100-99$ & $99-87.5$ & $87.5-75$ & $75-62.5$ & $62.5-50$ & $50-37.5$ & $37.5-25$ & $25-12.5$ & $12.5-0$ \\
\hline
\end{tabular}

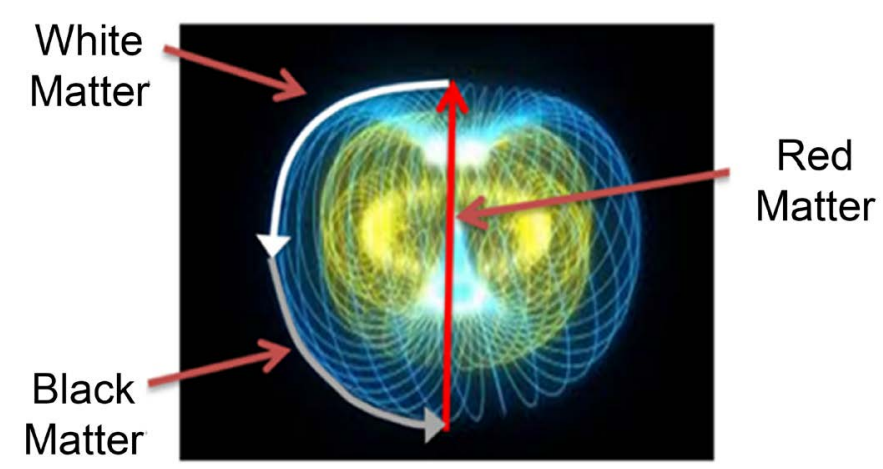

Figure 3. I particle vibration.

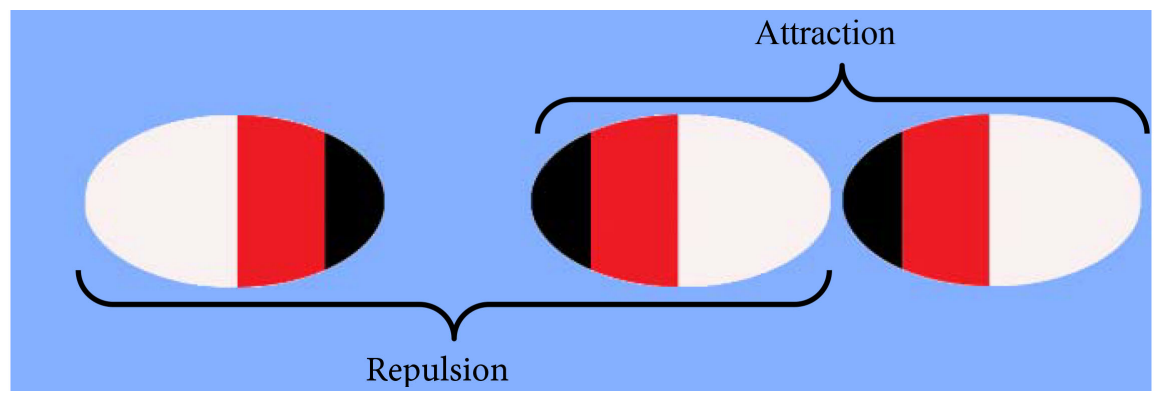

Figure 4. I-particles in attraction or repulsion state.

So we can write:

$$
S_{S_{\infty}}>S_{A_{1}}
$$

The I-Theory clearly shows that Entropy is the characteristic of repulsion.

Since Negentropy is the opposite force of Entropy, we can say that Negentropy is the characteristic of attraction. Negentropy starts from $A_{1}$ particles (Figure 5).

We already explained that an I-particle is electrically polarized, the vibration of the I-particle (its energy) is an electromagnetic wave. It also could be assimilated to a basic packet of information. The number of I-particles in repulsion or attraction state will change the nature of information. In the same way as binary code, the location of the I-particle in attraction state will also change the information. Therefore, we can say that Shannon Entropy is the description of the arrangement of I-particles. Maximum information is available in the Space element.

\subsection{I-Theory and Negentropy-The Concept of Intelligence}

In I-Theory, Negentropy is also called "Intelligence”. Intelligence is Red Matter. 


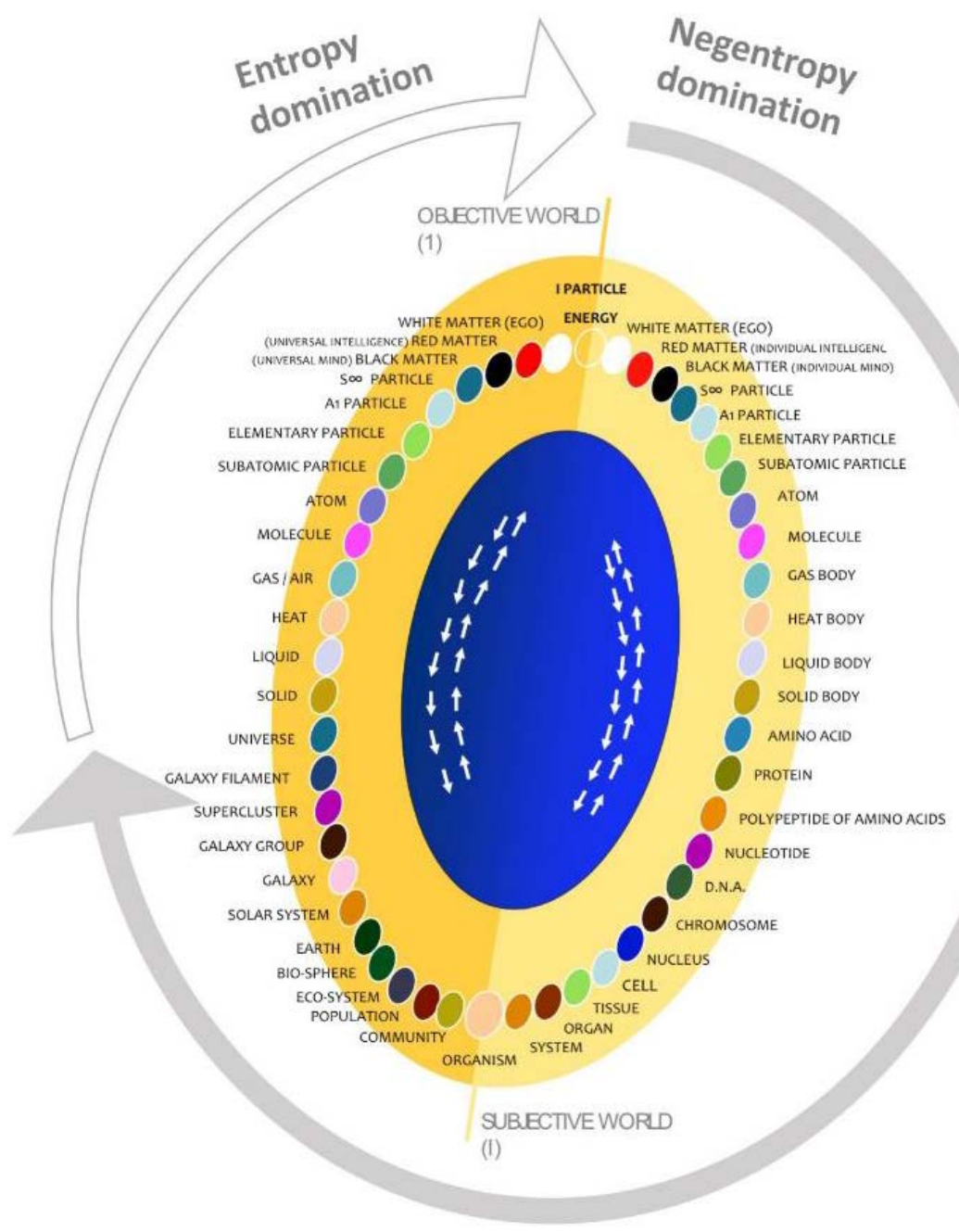

Figure 5. Entropy and negentropy domination

The word "Intelligence" has been chosen because the Universe is the manifestation of the energy pattern of two states:

- the illogical state (Entropy) creates disorder;

- the logical state (Intelligence) creates order.

Logic could not exist without Intelligence. Intelligence must be there for ordering elementary particles in the Universe.

As we state in Section 2.2, Negentropy is related to the free Entropy. Therefore, Negentropy is another kind of Entropy.

The I-Theory explains that:

- Negentropy is the feature of Gross space and from there Gross matter is formed;

- Entropy is the feature of Subtle space and from there Subtle matter is formed. Note: Gross matter is defined as matter with constituents of atoms and larger (molecule, cells, etc.), while Subtle matter is subatomic particles and smaller (elementary particles, I-particle).

The natural state of irregular I-particles is known as Entropy in modern 
science. The irregular energy pulses become arranged into a primary level known as Space. The dominating energy is repelling. When gravitation or attractive energy starts to create more order, a new dimension is formed known as Creative intelligence (Negentropy).

The repulsive forces of the Space element forming into Intelligence act as the source of creation. Even a fraction of a variation in the degree of the arrangement will bring changes in property or quality. Energy matter with maximum frequency is broadly called Black Matter (Strong force).

In the same pattern, White Matter and Red Matter manifest dimensions of energy. When the "I" vibration of repulsive force attains order, the dimension of repulsive force with White Matter domination is known as Subtle Space. The dimension with Black Matter domination is called Gross Space.

The $S_{\infty}$ particle is the term used to describe the arrangement of I-particles responsible for the creation of Space. Every object in this universe originates from this $S_{\infty}$ particle. In other words, the repulsive I-particle changes position so that its positive and negative come face to face in attraction, and the consequent dimension occurs.

In short, if Space (repelling Energy) is reduced, Negentropy dominates. If Space is increased, Entropy dominates.

The I-Theory predicts 2 kinds of Space: Gross Space and Subtle Space. Both are repelling energy. Gross Space is defined as when the portions of the I-particles' Black Matter are facing together, while Subtle Space is defined as when the portions of I-particles' White Matter are facing together.

White Matter is defined by low frequency (or high wavelength), and the space between I-particles forming Subtle space is higher than Gross Space's. So, we can say that repelling energy is less for Gross Space; in other words attraction is increasing. Therefore, Negentropy is a Gross Space feature and Entropy is a Subtle Space feature (Figure 6).

Entropy is one side of life and Intelligence is the other side. This can be summarized with Figure 7.

As shown in Figure 8, balanced Intelligence is pure Red Matter. Creative Intelligence is Black-Matter-dominated Red energy. Entropy, or "destructive Intelligence", is White-Matter-dominated Red energy.

In terms of the I-Theory, Red Matter that exists in a balanced frequency in the I-particle, the basic vibration, is the Intelligence behind the information of all objects in the Universe. When it is Black Matter dominated, that creative energy forms the gross world and Black energy-based Ignorance. We commonly call this process creation.

When the frequency vibration of Intelligence energy is lower, it is White Matter dominated. That White-Matter dominated creative energy forms the subtle world. We commonly call this process destruction, although nothing is destroyed except gross forms. Subtle forms dominated by White Matter are created. They reach the transparency of White Matter energy. 


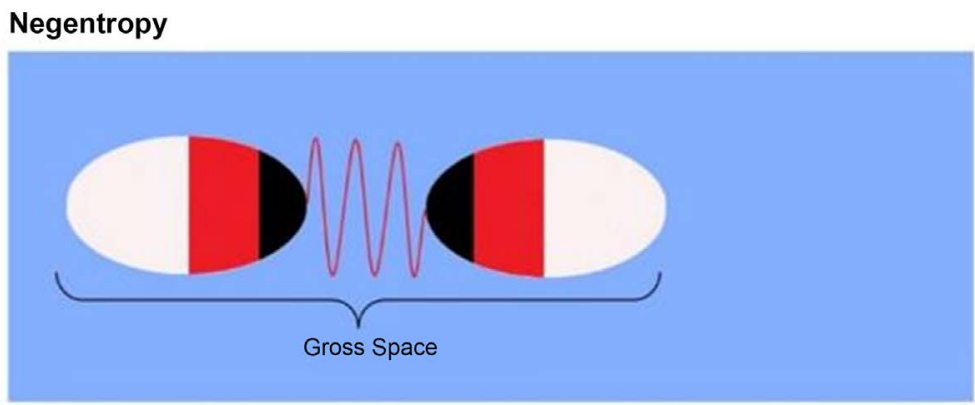

\section{Entropy}

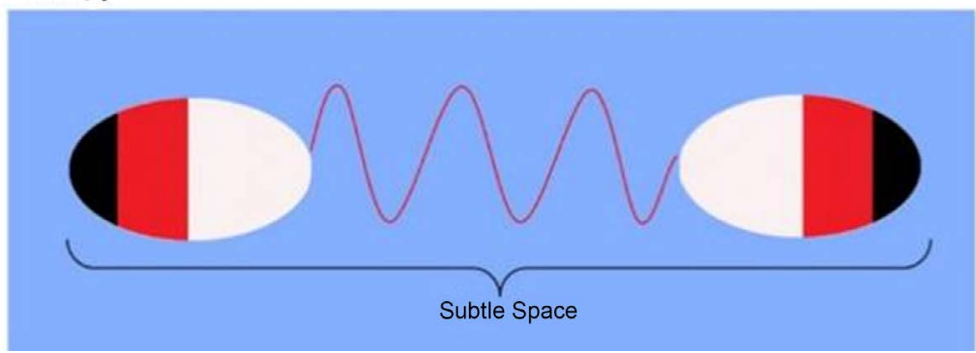

Figure 6. Entropy and negentropy as subtle and gross space.

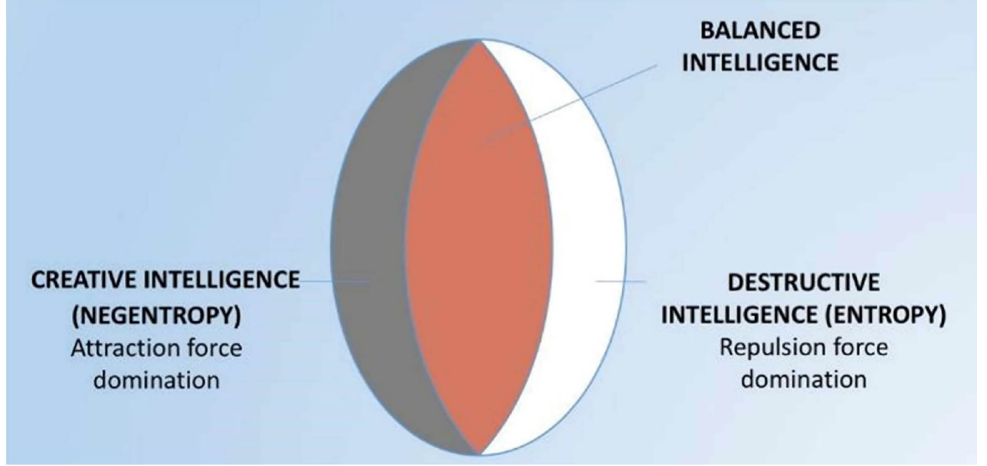

Figure 7. Different kinds of intelligence.

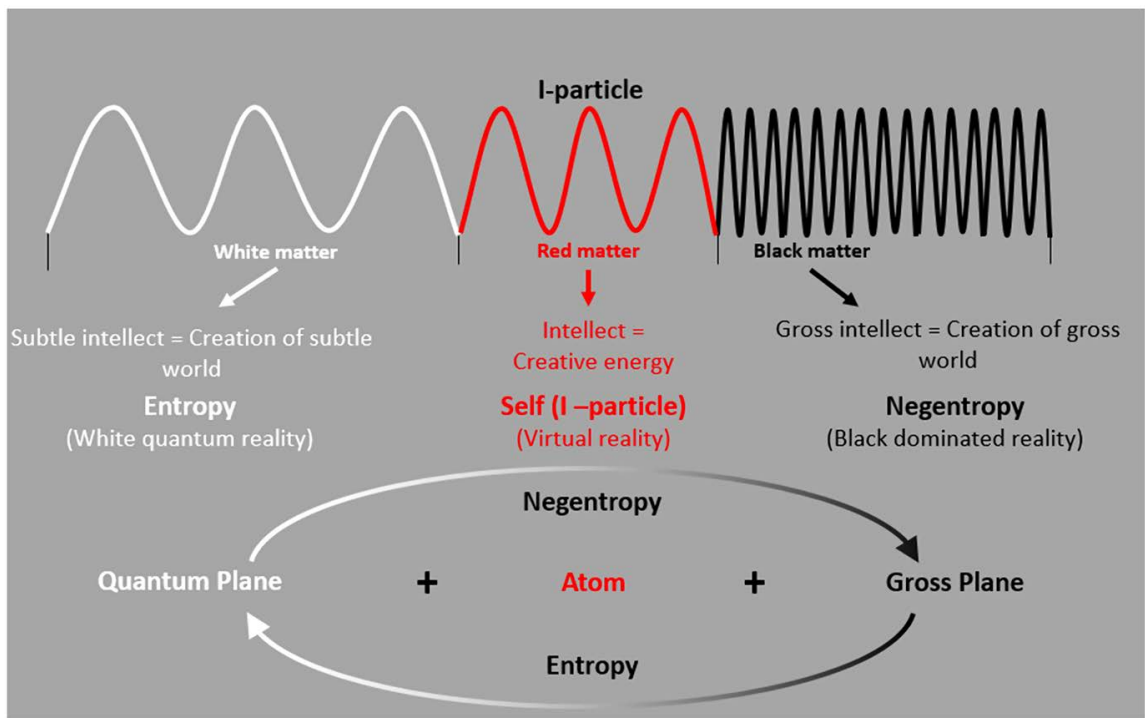

Figure 8. Different properties of Intelligence according to matter domination. 
In the I-Theory, each Matter is related to a fundamental interaction force [1]:

- Red Matter is associated with Gravity. It is pure gravitation force. It is Balanced Intelligence.

- White Matter is associated with Weak force. White Matter is attraction force coming from gravitation minus repulsion force between I-particles. It is Destructive Intelligence.

- Black Matter is associated with Strong force. Black Matter is attraction force coming from gravitation plus attraction force between I-particles. It is Creative Intelligence.

- Electromagnetic force is given by the arrangement between I-particles.

So we can say that Entropy is driven by the Weak Force (this is why it is called Destructive Intelligence) and Negentropy is driven by the Strong Force (the reason it is called Creative Intelligence).

Modern science describes creation in the following manner (Figure 9):

When time was Nil (0), Space, Time and Energy originated. At $10^{-43}$ seconds, gravity radiation occurred and at $10^{-34}$ seconds, the Universe considered as a vacuum began to expand very fast. At $10^{-30}$ seconds, fast expansion ended and atoms came into being from the state of nothingness. In $10^{-11}$ seconds, an electronic force split into electromagnetic force and weak nuclear force. At that time there were only quarks. At $10^{-6}$ seconds, 3 quarks each joined to form protons and neutrons, and at 3 minutes 42 seconds hydrogen, proton and neutron combined to form a hydrogen and helium nucleus. Hydrogen and helium acquired their own stability and order, and after an hour the nuclear process ended. After one year, the temperature of the Universe reduced and electrons made their entry and started circling the nucleus. At $10^{6}$ years, primordial galaxies and stars took shape. At $10^{9}$ years galaxies and stars occurred. While maintaining stability, Hydrogen and Helium were transformed into elements with complex atomic structure. Simple Hydrogen atoms formed other weight atoms like Uranium and Plutonium.

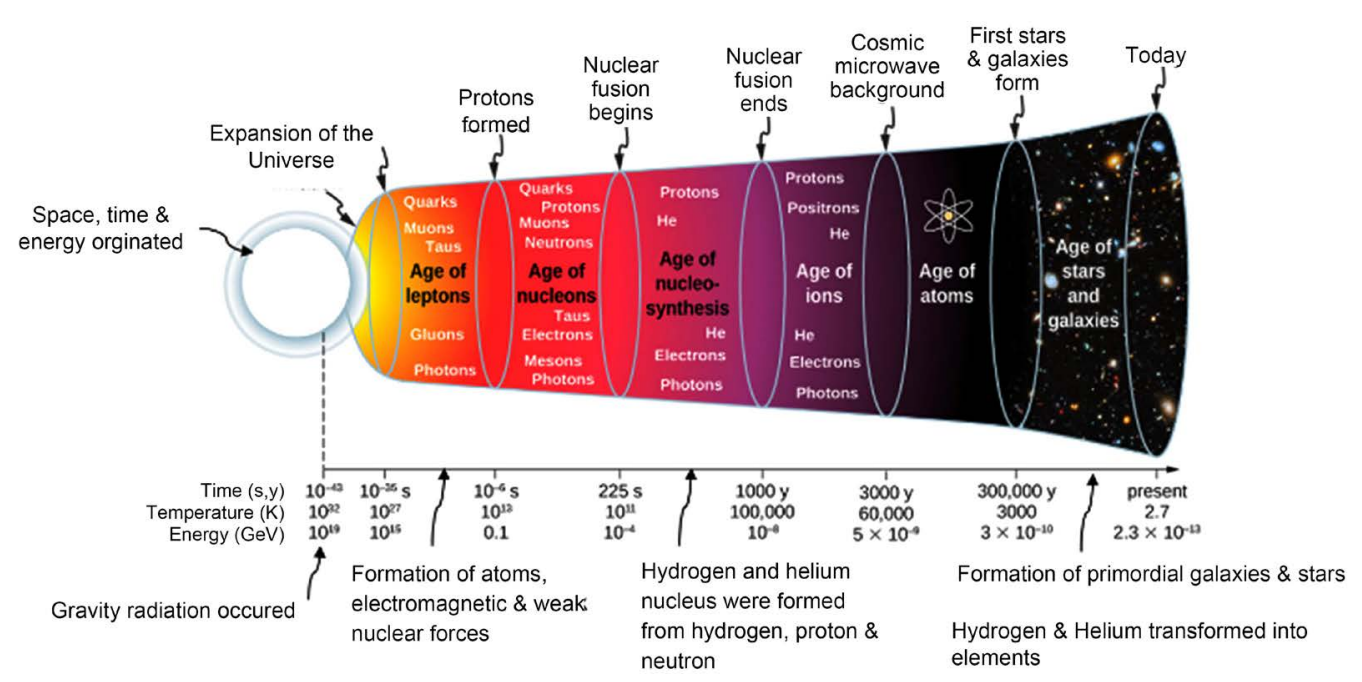

Figure 9. Creation of the universe [19]. 
At the beginning of the Universe, Gravity (Red Matter) started. At this time, Space was very less and Negentropy (Intelligence) dominated. Then Weak Force appeared. Weak Force is given by White Matter [1]. Its characteristic is repulsion or Entropy. Space suddenly increased. This is known as the Big Bang. After a short period, Strong Force appeared. Strong Force is given by Black Matter [1]. Its characteristic is attraction or Negentropy. Subtle space was destroyed and Gross elements were formed. Then Gross elements arranged themselves in order to create more complex structures.

\subsection{Entropy and Negentropy in Life Evolution Process}

Solar systems and galaxies were formed 4600 million years ago. Then the Earth cooled down and the pulsing of life began 3800 million years ago. Biological and non-biological evolutions from the elements finally resulted in single cell organism algae to bacteria and from bacteria to complex organisms. Cells came into existence 3600 million years ago, plant cells occurred 1200 million years ago, fish and worms appeared 530 million years ago, and vertebrates were seen 500 million years ago. Life spread from sea to land 440 million years ago. Insects appeared on land 400 million years ago, vertebrates on land came into existence 360 million years ago and 200 million years ago appeared the first mammal on land. Horses appeared 50 million years ago, dogs 30 million years ago, and monkeys and chimpanzees 20 million years ago. Original ancestors of man appeared 1.8 million years ago and the present day human, Homo sapiens, is traced back to 200,000 years (Figure 10).

In the evolution of matter and life, a force works against Entropy. Consider the birth of a baby. Ovum and sperm fuse to form a single cell, which multiplies by cell division and finally develops bone, muscle, teeth, and nail and becomes a miniature human being. The genes contain DNA, the Intelligence that controls

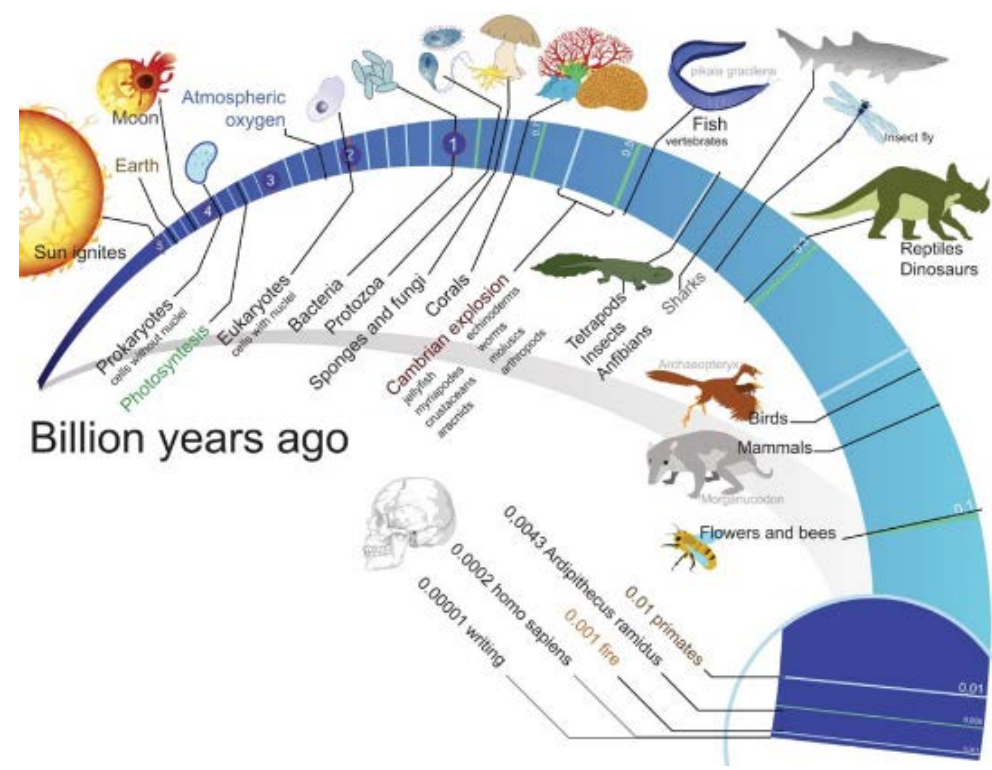

Figure 10. Life evolution [20]. 
the genetic imprint of an individual, which is present in the original cell. Similarly, the cosmic Intelligence keeps the Universe united.

The origin of Energy, and the complex cosmic growth, is because of the Intelligence behind it. Intelligence is a synonym of creativity. Order is made from disorder. Intelligence shapes particles of form and give life to it.

When Entropy has the upper hand, Intelligence fades away and disorder reigns, this conflict between these two forces has been going on since the beginning of creation.

The difference between Intelligence (or Negentropy) and Entropy is the period. The forming period is due to Intelligence, while the growing period is Entropy. For example, the birth of a baby is the victory of the great Intelligence. Infancy, childhood, youth, and old age mark the victory of Entropy. It is the same for galaxy, planets, and other objects.

In Nature, creation and destruction co-exist. When proteins are produced from amino acids in our body, it is creation. During digestion, sugar is burned to produce energy and this is destruction. Life cannot exist without destruction.

In the human begins life from a single cell, by cell division turns two, four, eight and finally becomes the individual sporting billions of cells.

It is the energy in DNA that creates varied traits in a single cell. Creation becomes possible by the energy in Entropy that goes through a constant process of change. The unique basic factor in nature and bio-life is the vibrating energy in frequency and wavelength. Even a fraction of a variation in angle creates changes in energy, and hence each variation has its own entity.

The DNA of each cell contains the blueprint of its information. It is preserved by the Intelligence which is Red Matter (or Gravitation Force). This Intelligence is seen in both growth and decay.

One gross form turns into another one when dominated by Black Matter (Strong Force). This is usually called destruction. A house crumbles and becomes sand. That sand is made into bricks and becomes another house.

Since Negentropy is Black-Matter Energy oriented and Black Matter is negatively electrical charged, one way for assessing Negentropy on the bio-plane is to measure electrical charge.

Nature has created the perfect ambience, which is a balance of Black and White Matter or Strong and Weak forces. A balanced state of White Matter energy and Black Matter energy is what we call stability, sustainability and harmony. The natural rhythm is maintained. When this equilibrium is lost, diseases occur.

\section{Conclusions and Perspectives}

The I-Theory gives a deeper perspective to Entropy and Negentropy and its implications for the universal cycles and life itself. In summary, Entropy is characterized by the repulsion force, while Negentropy is driven by attraction force. By introducing the $S_{\infty}$ particle as the Space particle (100\% of repulsion), we can de- 
fine Entropy and Negentropy as Subtle Space and Gross Space. The I-Theory predicts that attraction force is starting from $A_{1}$ particle (or Higgs Boson). Therefore, Negentropy is starting from that particle.

In this article, we have defined the Creative Intelligence as Negentropy and the Destructive Intelligence as Entropy.

Finally, we have shown that Entropy is driven by the Weak Force as well as Negentropy is driven by the Strong Force.

\section{Conflicts of Interest}

The authors declare no conflicts of interest regarding the publication of this paper.

\section{References}

[1] Isa Swami, H.H. and Dumas, C. (2019) I-Theory: A Unifying Quantum Theory? Journal of High Energy Physics Gravitation and Cosmology, 5, 332-359. https://doi.org/10.4236/jhepgc.2019.52019

[2] Carnot, S. (1824) Réflexions sur la puissance motrice du feu et sur les machines propres à développer cette puissance. Bachelier Libraire, Paris.

[3] Clausius, R. (1865) On Various Easily Applicable Forms That Can Be Given to the Fundamental Equations of the Mechanical Theory of Heat.

[4] Boltzmann, L. (1877) Sur la relation entre le second principe de la thermodynamique et la théorie des probabilités, en rapport avec l'équilibre thermique.

[5] Hawking, S. (1975) Particle Creation by Black Holes. Communications in Mathematical Physics, 43, 199-220. https://doi.org/10.1007/BF02345020

[6] Shannon, C. (1948) A Mathematical Theory of Communication. Bell System Technical Journal, 27, 379-423, 623-656. https://doi.org/10.1002/j.1538-7305.1948.tb00917.x

[7] Schrodinger, E. (1944) What Is Life? The Physical Aspect of the Living Cell. Dublin Institute for Advanced Studies, Dublin.

[8] Brillouin, L. (1953) Negentropy Principle of Information. Journal of Applied Physics, 24, 1152-1163. https://doi.org/10.1063/1.1721463

[9] Brillouin, L. (1959) La science et la théorie de l'information, Masson.

[10] Gibbs, W. (1873) A Method of Geometrical Representation of the Thermodynamic Properties of Substances by Means of Surfaces. Transactions of the Connecticut Academy, 2, 382-404. https://doi.org/10.1093/nq/s4-XII.307.382

[11] Massieu, M.F. (1869) Sur les fonctions caractéristiques des divers fluides. Compte rendu Académie des Sciences, 69, 858-862.

[12] Massieu, M. (1869) Addition au precedent memoire sur les fonctions caractéristiques. Compte Rendu Académie des Sciences, 69, 1057-1061.

[13] Planck, M. (1945) Treatise on Thermodynamics. Dover, New York.

[14] Planes, A. and Vives, E. (2002) Entropic Formulation of Statistical Mechanics. Journal of Statistical Physics, 106, 827-850. https://doi.org/10.1023/A:1013778810460

[15] Scheilman, J.A. (1997) Temperature, Stability, and the Hydrophobic Interaction. Biophysical Journal, 73, 2960-2964. https://doi.org/10.1016/S0006-3495(97)78324-3 
[16] Hens, Z. and Hemptinne, X.D. (1996) Non-Equilibrium Thermodynamics Approach to Transport Processes in Gas Mixtures. Trends in Research, 13-21. arXiv:chao-dyn/9604008

[17] Xu, B., et al. (2019) Comprehensive Evaluation of Basic Public Health Services in Nanning City Based on Entropy Weight TOPSIS Method. Practical Preventive Medicine, 26, 156-159.

[18] Chen, J. (2019) Aquatic Ecosystem Health Assessment of a Typical Sub-Basin of the Liao River Based on Entropy Weights and a Fuzzy Comprehensive Evaluation Method. Scientific Reports, 9, Article No. 14045.

https://doi.org/10.1038/s41598-019-50499-0

[19] https://cnx.org/contents/ePrDJvxu@1.19:bIMtPPGL@7/11-7-Evolution-of-the-Earl y-Universe

[20] https://commons.wikimedia.org/wiki/File:Timeline evolution of life.svg 